\title{
Kompetenznetz Depression, Suizidalität
}

$\mathrm{D}$ epression ist eine Erkrankung mit besonderer medizinischer und gesundheitspolitischer Brisanz. Diese Brisanz ergibt sich aus drei Faktoren:

- Die von der WHO in Auftrag gegebene Studie „Global Burden of Disease“ (1997) kommt zum Ergebnis, dass Depression wie kaum eine andere Krankheit die Betroffenen beeinträchtigt und zu den wichtigsten Ursachen für vorzeitigen Tod gehört. Diese Problematik wird sich nach Prognosen der WHO in der Zukunft weiter verschärfen.

- Viele Betroffene suchen keine ärztliche Behandlung auf oder werden von (haus)ärztlicher Seite nicht als depressiv erkannt. Es gibt derzeit massive diagnostische Defizite.

- Obwohl mit Antidepressiva und Psychotherapie wirksame Behandlungsoptionen zur Verfügung stehen, kommen diese zu selten zur Anwendung. Schätzungen gehen davon aus, dass lediglich 10\% der Patienten mit Depressionen eine adäquate Akutbehandlung erhalten. Hinsichtlich der symptomsupressiven und rückfallverhütenden Langzeitbehandlung dürfte die Situation noch problematischer sein.

Vor dem Hintergrund dieser beunruhigenden Situation wurde nach einem 1 1/2 jährigen Beantragungsprozess und Auswahlverfahren durch internationale Gutachter im Juli 1999 das Kompetenznetz „Depression, Suizidalität gestartet. Gefördert wird dieses Projekt über 5 Jahre im Rahmen des BMBF-Forschungsprogramms „Kompetenznetze in der Medizin“. Ziel dieses Forschungsprogramms ist eine bessere Kooperation und Abstimmung der forschenden Einrichtungen (horizontale Vernetzung). Zudem wird eine bessere Zusammenarbeit zwischen den verschiedenen Versorgungsebenen von Hochschulen bis hin zur Primärversorgung angestrebt (vertikale Vernetzung). Durch das „Zuckerbrot“ der finanziellen Förderung wurde bereits in der Planungsphase ein intensiver Abstimmungsprozess in Gang gesetzt, der zu zahlreichen stabilen Vernetzungen bisher getrennt arbeitender Bereiche in der Forschung und Versorgung depressiver Patienten geführt hat. Eingebunden in das Kompetenznetzwerk sind Universitätskliniken, ferner Bezirks- und Landeskrankenhäuser, psychosomatische Kliniken, niedergelassene Ärzte und Praxisnetze,
Krankenkassen, die Betroffenen und weitere wichtige Partner im Versorgungssystem.

Nach der Zeit des Säens und Pflanzens beginnt nun die Erntezeit und eine Auswahl der ersten Früchte wird in den Beiträgen dieses Schwerpunktheftes vorgestellt. Sie dokumentieren die Breite der Forschungsthemen des Kompetenznetzes „Depression, Suizidalität“, die von der Molekulargenetik bis zur Versorgungsforschung reicht.

Neben hochkarätiger Forschung betreibt das Kompetenznetz „Depression, Suizidalität“ eine intensive Öffentlichkeitsarbeit mit dem Ziel, auch über diesen Weg das diagnostische und therapeutische Defizit zu reduzieren und eine bessere Interessensvertretung der Betroffenen in gesundheitspolitischen Entscheidungsprozessen zu erreichen. Ein Baustein ist die Homepage (www.kompetenznetz-depression.de) mit täglich 1.500 Besuchern, auf der Informationen und andere Hilfestellungen für Betroffene und weitergehende Informationen zum Kompetenznetz zur Verfügung stehen.
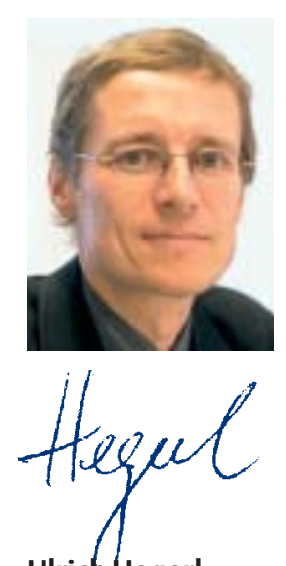

UlrichHegerl

Sprecher des Kompetenznetzes „Depression, Suizidalität“ Psychiatrische Klinik der Ludwig-Maximilians-Universität München 